\title{
Consideraciones para la incorporación de la Inteligencia Artificial en un programa de pregrado de Ingeniería Eléctrica
}

Considerations for Introducing Artificial Intelligence in an Electrical

Engineering undergraduate program

\author{
Volumen 21, Número 2 \\ Mayo - Agosto \\ pp. 1-25
}

Marvin Coto Jiménez

Citar este documento según modelo APA

Coto Jiménez, Marvin. (2021). Consideraciones para la incorporación de la Inteligencia Artificial en un programa de pregrado de Ingenería Eléctrica. Revista Actualidades Investigativas en Educación, 21(2), 1-25. Doi. 10.15517/aie.v21i2.44893 


\title{
Consideraciones para la incorporación de la Inteligencia Artificial en un programa de pregrado de Ingeniería Eléctrica Considerations for Introducing Artificial Intelligence in an Electrical Engineering undergraduate program
}

\section{Marvin Coto Jiménez ${ }^{1}$}

Resumen: Con la rápida proliferación de técnicas y aplicaciones, la inteligencia artificial ha adquirido gran relevancia en diversos campos de la sociedad, lo cual incluye nuevas formas de resolver problemas relacionados con sistemas de energía, de señales y de información, los cuales son analizados dentro de una carrera como Ingeniería Eléctrica en la Universidad de Costa Rica. Dada la relevancia del tema, algunos autores han señalado la importancia de difundir el conocimiento de la Inteligencia Artificial más allá de laboratorios especializados y programas de posgrado donde usualmente se han desarrollado. Este ensayo tiene como objetivo aportar a la discusión sobre la conveniencia y forma apropiada de introducir la inteligencia artificial en el currículo de pregrado en carreras de ingeniería, en especial en un programa de estudio de ingeniería eléctrica de la Universidad de Costa Rica, en el cual se propicia una formación general. Una propuesta como esta debe tener en cuenta las bases con que cuentan las personas discentes, así como los contenidos y estrategias convenientes para lograr una introducción adecuada en su formación académica y el beneficio de la profesión. En el ensayo se presentan y discuten estas consideraciones a la luz de la conceptualización de la inteligencia artificial y su aplicabilidad en la actualidad de la ingeniería.

Palabras clave: educación superior, inteligencia artificial, ingeniería eléctrica, tecnología.

\begin{abstract}
With the rapid proliferation of techniques and applications, artificial intelligence has acquired great relevance in our society, including new ways of solving problems related to energy, signal, and information systems, which are studied in a career such as Electric Engineering. Given the relevance of the topic, some authors have pointed out the importance of spreading the knowledge of Artificial Intelligence beyond specialized laboratories and graduate programs where usually have been developed. This essay aims to contribute to the discussion about the convenient and appropriate way of introducing the teaching of artificial intelligence at undergraduate levels in engineering careers, especially in an electrical engineering study program at the University of Costa Rica, which encourages general training. The proposal must take into account the bases that the students have, as well as the contents and convenient strategies to achieve a suitable introduction in their academic training and the benefit of the profession. In the essay, these considerations are presented and discussed in light of the conceptualization of artificial intelligence and its applicability in engineering today.
\end{abstract}

Keywords: higher education, artificial intelligence, electrican engineering, technology.

\footnotetext{
1 Universidad de Costa Rica, en la Escuela de Ingeniería Eléctrica, San José, Costa Rica. ORCID: https://orcid.org/0000-0002-6833-9938
}

Dirección electrónica: marvin.coto@ucr.ac.cr

Ensayo recibido: 18 de noviembre, 2020

Enviado a corrección: 18 de marzo, 2021

Aprobado: 19 de abril, 2021 


\section{Introducción}

Para iniciar el análisis del tema de inteligencia artificial (abreviado comúnmente como I.A.) y su introducción en programas de pregrado, es importante precisar algunas definiciones, y de esta manera facilitar la comprensión y contextualización de la materia en cuestión. Como punto de partida se debe mencionar que la propia conceptualización encuentra una dificultad inicial en el concepto más importante al que hace referencia: la inteligencia artificial, ya que sobre esta no existe un consenso. En sus inicios, como una rama independiente del conocimiento, en la década de 1950 en los Estados Unidos de América, se identificó con conjeturas como: "si todo aspecto del aprendizaje o cualquier otra característica de la inteligencia puede ser en principio descrita con precisión, entonces se puede construir una máquina que la simule". Esta afirmación está contenida en una publicación de 1995 de McCarthy, Minsky, Rochester y Shannon, pioneros de esta disciplina (Müler y Bostrom, 2016). Con este tipo de intuiciones sobre el aprendizaje y capacidades de la inteligencia humanas, se pensó que podrían replicarse fuera de la mente y realidad biológica humana usando máquinas.

Uno de los autores pioneros en el tema, Marvin Minsky, formuló una definición frecuentemente citada: "La inteligencia artificial es la ciencia de hacer que las máquinas realicen tareas que requerirían inteligencia si fueran realizadas por seres humanos" (Rubio, Lermanda, Segura, Martínez y Vidal, 2018, p. 451).

Uno de los problemas con esta conceptualización del término, y otras presentadas a lo largo de las últimas décadas, es que involucran la palabra "inteligencia". Sobre ésta se ha mantenido una amplia discusión de sus alcances, y su relación con el comportamiento o tareas que se pretenden emular en una máquina o computadora. Es por estas razones que uno de los mayores retos que existe en la definición de inteligencia artificial es lograr una conceptualización que no haga referencia a la inteligencia humana.

El diccionario de la RAE indica como definición del término: "Disciplina científica que se ocupa de crear programas informáticos que ejecutan operaciones comparables a las que realiza la mente humana, como el aprendizaje o el razonamiento lógico" (Real Academia Española, 2021). Esta definición, sin embargo, contempla una enorme cantidad de posibilidades dentro de las tareas que realiza la mente humana, algunas de las cuales no se contemplan necesariamente dentro de la disciplina, tales como el movimiento de extremidades y la regulación de procesos internos. 
La visión inicial de alcanzar una inteligencia fuera del cerebro humano, que pudiera realizar cualquier tarea, se conoce hoy como inteligencia artificial general. La mayoría de aplicaciones actuales de esta área del conocimiento se enfocan en resolver problemas específicos dentro de áreas como la ingeniería y las ciencias. Solamente una pequeña parte de investigadores cree que se acerca el momento de tener como un objetivo una inteligencia artificial general (Müler y Bostrom, 2016, p. 2).

Con el desarrollo logrado en las últimas décadas, la necesidad de conceptualizar la disciplina con claridad ha sido evidente. Autores como Russell y Norvig (2002) han sistematizado las formas de definir la inteligencia artificial en cuatro categorías, representadas usando cuadrantes. Los cuadrantes están delimitados por ejes de pensamiento humano y racional, y acción inteligente y racional. Dentro de este cuadrante, la definición previa de Minsky podría ubicarse en el eje "actuar como las personas", ya que se trata de una imitación de comportamiento.

Todas las diferentes tendencias conceptuales que se puedan establecer alrededor del tema remiten a estrategias de investigación particulares. Por ejemplo, las definiciones en el eje "actuar de forma racional" llevan a la definición de entidades (agentes) que pueden percibir su entorno y operar de forma autónoma. La investigación y desarrollo se dirige en este caso al estudio de estos agentes, los cuales pueden ser desde programas de computadora hasta elementos físicos, como robots.

Para el presente ensayo se enmarca el concepto de inteligencia artificial dentro de la categoría de "actuar como las personas", dado que se identifica con un enfoque apto para su incorporación dentro de una carrera de ingeniería, especialmente a nivel de pregrado. Como indica Kok, Boers, Kosters, Van der Putten y Poel (2009, p. 2), una definición enciclopédica del idioma inglés tiene entre sus acepciones para la inteligencia artificial "la extensión de la inteligencia humana a través del uso de computadoras, así como en el pasado la fuerza física fue extendida con el uso de herramientas mecánicas". Esta noción de imitación del comportamiento humano inteligente es la que representa un mayor interés para el presente trabajo.

Hacer énfasis en el hecho de la inteligencia artificial trata de una imitación del comportamiento humano inteligente, permite poner la atención en la resolución de problemas específicos. En estos problemas la inteligencia humana puede conceptualizar y definir el problema, pero su capacidad no permite resolver o realizar la tarea de forma eficiente. Por 
esto se utilizan las computadoras, con las cuales se extienden las capacidades humanas, imitando su comportamiento inteligente.

Por ejemplo, el uso de la lógica es una de las facultades del cerebro humano con las cuales se toman muchas decisiones, y es un claro componente fundamental de la inteligencia humana (Copeland 2015, p.7). Con la lógica se obtienen conclusiones a razonamientos, incluso, de forma abstracta, como el siguiente:

Si " $x$ " o " $y$ " son verdad, $y$ dado que sabemos que " $y$ " es falso, entonces se sigue que " $x$ " es verdadero.

Es posible procesar este tipo de argumentos en una computadora con una velocidad muy superior a la del cerebro humano. $Y$ es con principios como este, junto con una cantidad creciente de algoritmos desarrollados, que las implementaciones de sistemas basados en inteligencia artificial han permitido concretar una serie de aplicaciones que ya forman parte del entorno de empresas, investigaciones y de la vida cotidiana de muchas personas. Entre estas aplicaciones se encuentran (Das, Dey, Pal y Roy, 2015, p. 32):

1.Organización automática de noticias dependiendo de su contenido: A partir de la información del texto se extraen etiquetas de contenido y su relación con otros contenidos.

2.Sistemas de recomendación en sitios de internet de ventas: Las búsquedas de las personas usuarias, así como el interés manifestado en la información a la que acceden permiten hacer predicciones sobre los productos o servicios que estas personas podrían adquirir.

3. Sistemas de apoyo al diagnóstico médico a partir de análisis automático de imágenes.

4. Reconocimiento de voz en dispositivos celulares inteligentes y en computadoras. Tanto el problema del reconocimiento de voz como el de análisis automático de imágenes tienen en común la clasificación de información variable y nunca repetida (por ejemplo, todas las personas tienen una voz única y pronuncian las palabras de formas variables).

Algunas de estas aplicaciones pueden ubicarse dentro de áreas que se estudian e investigan en la Ingeniería Eléctrica, entiendo esta como una rama de la ingeniería que hace uso de "las matemáticas, la física, las técnicas de análisis de sistemas eléctricos, electrónicos, electromagnéticos y computacionales para obtener beneficios a partir de la energía eléctrica, las señales y la información." (Escuela de Ingeniería Eléctrica, s.f., párrafo 4). En términos de delimitación, la definición de los alcances del área de Ingeniería Eléctrica también representa 
un reto significativo, dado el dinamismo del área y del rápido surgimiento de nuevas posibilidades de aplicación de la electrónica, los sistemas de energía, las telecomunicaciones, la fotónica, entre otras.

Para establecer una diferencia con las ciencias, y en particular las ciencias aplicadas, se identifica a la ingeniería con la síntesis, a diferencia de las ciencias, las cuales se centran en el análisis (Simon 2019, p. 4). Es decir, la ingeniería se centra en cómo diseñar o adoptar objetos materiales para que tengan propiedades deseadas.

Por ejemplo, si se desea que una computadora o un sistema cuente con la propiedad de reconocer imágenes, se analiza dentro del área de reconocimiento de patrones y se diseñan softwares y algoritmos para lograr este objetivo. La multiplicidad de problemas y subproblemas que se van generando, producen una gran cantidad de sub-áreas de especialidad en la Ingeniería Eléctrica. Por ejemplo, Dorf (2018) establece como pertenecientes al estudio de la Ingeniería Eléctrica los circuitos eléctricos, el procesamiento de señales (incluye acústica y procesamiento de habla), la electrónica (incluye la optoelectrónica), el electromagnetismo, la instrumentación y dispositivos eléctricos, los sistemas de energía, las telecomunicaciones, los sistemas digitales, la ingeniería en computadoras, los sistemas (incluyendo sistemas de control) y la ingeniería biomédica.

Dado que se toma como base para el presente análisis la carrera de Ingeniería Eléctrica de la Universidad de Costa Rica, se pueden señalar relaciones entre esta área de la ingeniería y la inteligencia artificial en investigaciones actuales, como el reconocimiento automático de imágenes, así como los procesos de reconocimiento de voz, los cuales se han incorporado en programas de estudio a nivel de posgrado, o en laboratorios de investigación dentro de Escuelas de Ingeniería Eléctrica, como en el caso del Laboratorio de Reconocimiento de Patrones y Sistemas Inteligentes (PRIS-Lab) de la Universidad de Costa Rica.

Hasta el momento, la introducción de los conceptos de la inteligencia artificial como un área propia y su relación con la carrera no se han considerado para formar parte del plan de estudios de ingeniería eléctrica. Con estos elementos se presentará para la discusión los contenidos apropiados para introducir el tema de inteligencia artificial dentro de un programa de pregrado, así como consideraciones sobre la evaluación y el uso de herramientas TIC exploradas en experiencias recientes documentadas en la literatura.

De esta manera, el propósito del ensayo es visibilizar la importancia de la temática de inteligencia artificial en su relación con la ingeniería eléctrica, y aportar a la discusión sobre la forma en que pueda ser incorporada en un currículo. 


\section{Desarrollo del tema}

La tesis central de este ensayo es que en un programa de pregrado de ingeniería eléctrica tiene cabida la introducción de la inteligencia artificial, dado que el estudiantado ha recibido fundamentos matemáticos y de programación que permiten apreciar, contextualizar y aplicar algunos algoritmos de esta área del conocimiento en problemas propios de ingeniería eléctrica. Como un segundo punto, la cantidad de aplicaciones y el potencial que tiene la inteligencia artificial en esta área del conocimiento hacen que esta introducción del tema sea relevante y necesaria. Sin embargo, dado que en la literatura no se cuenta con referencias que tengan el enfoque aplicado de la inteligencia artificial en la disciplina de Ingeniería Eléctrica, se debe realizar una valoración de contenidos y estrategia de introducción de los distintos temas.

En la Figura 1 se muestra, como referencia, el plan de estudios de la carrera de Ingeniería Eléctrica de la Universidad de Costa Rica, en el énfasis de Electrónica y Telecomunicaciones. Durante los primeros cuatro bloques se contempla una formación matemática considerable, con cursos de cálculo, ecuaciones diferenciales y matemática superior, así como de materias de física y programación.

Como referencia, los fundamentos matemáticos requeridos para desarrollar temas de inteligencia artificial son (Rawle, 2018):

- Análisis matemático: incluye transformaciones, álgebra lineal, funciones ortonormales.

- Métodos numéricos: La solución de problemas requiere frecuentemente de estrategias de optimización, procesos estocásticos y aplicación de heurísticas.

- Teoría de la probabilidad. 
Figura 1

Plan de estudios del programa de Ingeniería Eléctrica de la Universidad de Costa Rica

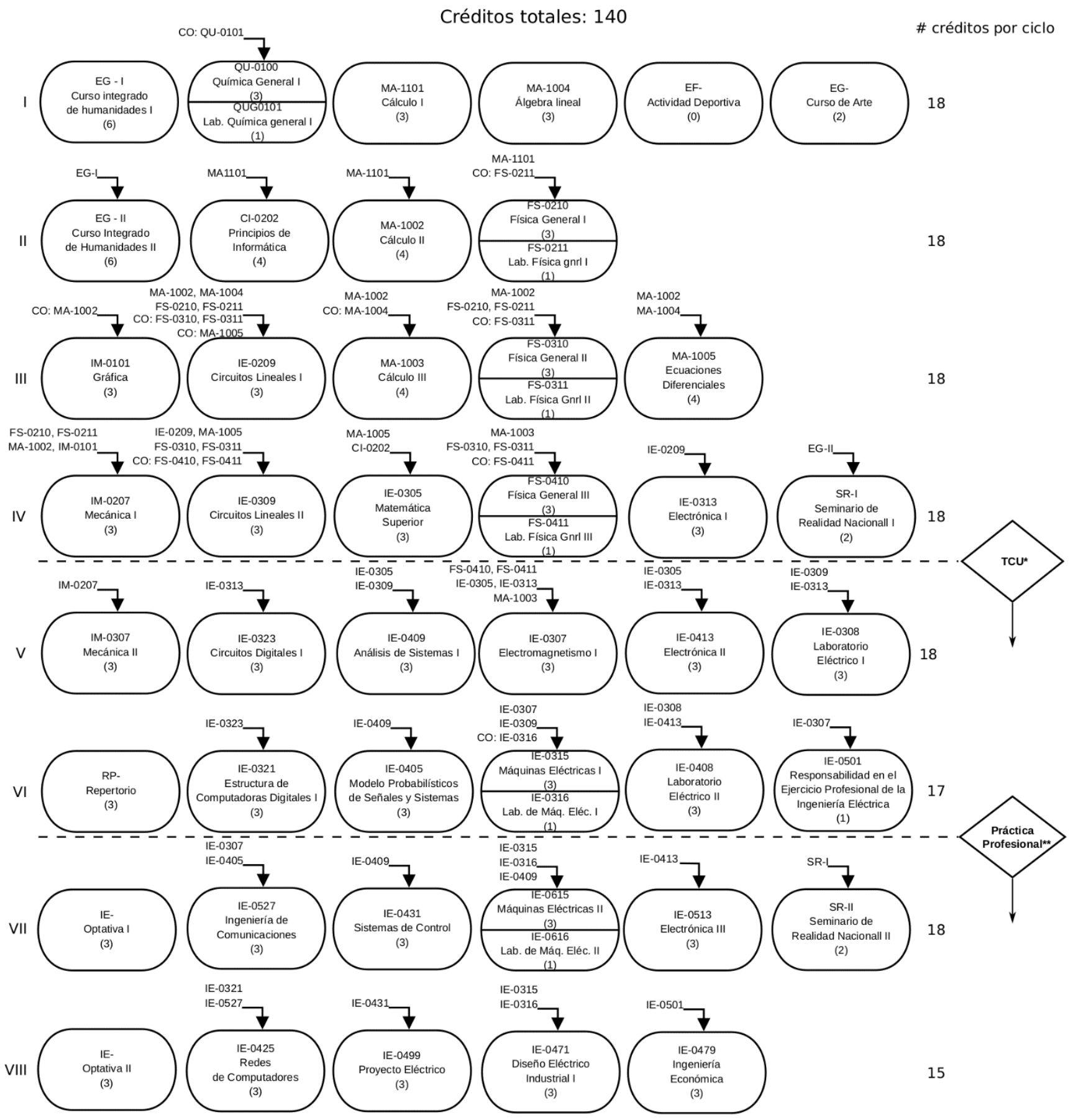

Fuente: Escuela de Ingeniería Eléctrica, Universidad de Costa Rica (2020, p. 1) 
Estos temas están cubiertos en los primeros seis bloques del programa de estudios en mención, lo cual representa los primeros tres años de carrera. Posiblemente se deba fortalecer algunos conceptos como heurísticas y métodos numéricos, pero los temas están al alcance del estudiantado.

De esta manera, se puede facultar en el estudiantado no solamente la adquisición de conocimiento, sino una visión aplicada que permita detectar problemas reales relacionados con inteligencia artificial en el ejercicio de la profesión. Estos problemas tienen en común que sus soluciones "resultan difíciles o impracticables por otros métodos" Miró-Juliá (2003, p. 1).

Es relevante también señalar que el mercado de trabajo en el área de inteligencia artificial es promisorio, dado el creciente número de aplicaciones prácticas que surgen constantemente en nuestros días (Liau y Guzide 2019). Por su parte, la selección de contenidos y de las estrategias de evaluación es de vital importancia para lograr una incorporación exitosa a los programas de estudio y al saber de esta disciplina.

Para la incorporación de la temática propuesta en un programa de estudio de ingeniería, también es importante considerar paradigmas y enfoques de aprendizaje en esta rama. Entre las opciones que han surgido se encuentran las STEM, un paradigma de enseñanza de ciencias, tecnología, ingeniería y matemática (acrónimo de Science, Technology, Engineering and Mathematics), para responder a las demandas y necesidades que en la actualidad tienen los estudiantes, las instituciones y el contexto socio y profesional.

Para estas demandas y necesidades, como indican Blackshields et al. (2016, p. 17), es necesario que los discentes "doten de sentido al flujo complejo y dinámico de información al que están expuestos y fomenten una comprensión profunda de la naturaleza de las interdependencias, espoleen la curiosidad y la indagación y sustenten la capacidad de desarrollar planes de vida".

Esto ha llevado a autores como Grinsztajn, Imperiale e Igarza (2019) a plantear procesos de innovación para

abordar el conocimiento de manera no fragmentada, encontrando los puntos de intersección de los conocimientos, su significatividad en relación con los problemas que el profesional deberá enfrentar, que difícilmente presentan el contenido en forma aislada, sino por el contrario, integran y entrelazan conocimientos provenientes de diversas disciplinas. (p. 4) 
En el caso de inteligencia artificial e ingeniería eléctrica, el proceso de innovación propuesto en un programa de estudios, y el abordaje indicado por estos autores tiene un requerimiento inicial en determinar los puntos de intersección entre ambos y la relación con los problemas que el profesional en ingeniería eléctrica deberá enfrentar.

\subsection{Proposición}

A partir de lo expuesto, se sostiene la propuesta de incorporar la inteligencia artificial en el currículo de pregrado de ingeniería, en particular en la carrera de Ingeniería Eléctrica. El estudiantado de esta carrera cuenta con el bagaje necesario de los fundamentos teóricos, y el tema reviste de suficiente relevancia y actualidad. Sin embargo, dado que la inteligencia artificial es un área del conocimiento creciente y cambiante, cuyos alcances y reflexiones están más allá del campo de acción de la ingeniería, es imperativo establecer una selección adecuada de contenidos y estrategias para su implementación exitosa en las aulas del pregrado. Adicionalmente, la introducción de contenidos debe contemplar también un énfasis en la parte ética de la inteligencia artificial, debido a las grandes implicaciones que podría tener a nivel global en un futuro cercano.

\subsection{Argumentos para la discusión}

La relevancia que tiene la inteligencia artificial en el desarrollo tecnológico ligado a temas de ingeniería eléctrica es una de las evidencias que podemos encontrar para justificar la pretensión de extender los alcances de la formación en esta área del conocimiento dentro de dicha carrera. Uno de los mayores retos de la propuesta es cómo extender estos alcances de una manera didáctica, con la cual se pueda equipar al estudiantado con recursos para la identificación, abordaje y solución de problemas del mundo actual (Mota-Valtierra, RodríguezReséndiz, y Herrera-Ruiz, 2019, párr. 1).

Los problemas que se están abordando a nivel de investigación, del ejercicio profesional y de la industria con herramientas de inteligencia artificial, tienen en común el ser dependientes "de un conjunto amplio de representaciones del conocimiento, de mecanismos de razonamiento, de algoritmos de búsqueda y de técnicas de aprendizaje" (Miró-Juliá, 2003, p.1). Por ejemplo, en la literatura se encuentran abundantes casos de aplicaciones de técnicas de inteligencia artificial en la gran mayoría de áreas de la ingeniería eléctrica presentadas en la Sección 1. Entre las publicaciones ya se encuentran algunas que realizan una revisión de la literatura actual en temas como los sistemas de energía (Ibrahim y Morcos, 2002), el 
equipamiento eléctrico (Han y Song, 2003) y el procesamiento de señales (Choong, , Reaz, y Mohd-Yasin, 2005).

En estos problemas, aspectos como la cantidad de información que se debe manejar, la dependencia de un conjunto amplio de variables y las relaciones mismas entre grandes cantidades de datos hacen que los sistemas basados en inteligencia artificial sea la opción por la que se decanten las y los profesionales en sus distintos ámbitos, dado el desarrollo de algoritmos adecuados y de la capacidad de cómputo actuales.

Por su parte, es notorio que, a pesar de la gran cantidad de publicaciones que relacionan ambas áreas del conocimiento, no se encuentran referencias en forma de libros de texto dirigidos específicamente a estudiantes o especialistas de Ingeniería Eléctrica. En términos de diseño de un curso introductorio de inteligencia artificial en un programa de pregrado de ingeniería eléctrica, se plantea entonces la necesidad de organizar la abundante información para que pueda tener un sentido de unidad y a la vez de aplicación para que el estudiantado pueda obtener una formación adecuada.

La idea de permear programas de pregrado con la formación en la inteligencia artificial no es nueva, ya que como indican Liao y Guzide (2019), se puede identificar una tendencia hacia la apertura de cursos introductorios sobre inteligencia artificial en programas de pregrado universitarios. Sin embargo, indican los mismos autores, el planteamiento de un curso general no se puede considerar adecuado por sí mismo para satisfacer la formación necesaria en esta materia.

En cambio, se hace necesario idear mejores estrategias para lograr la integración de los conocimientos de una mejor manera en el curriculum del estudiantado. Uno de los problemas que conlleva la pretensión de generalidad es la gran variedad de temas que están contenidos dentro del área de inteligencia artificial, tanto por los tipos de problemas que son abordados (de búsqueda, de optimización, de clasificación, agrupamiento, regresión), como por las familias de algoritmos que cada uno de estos problemas contempla como posibles caminos de solución. Como señalan DeNero y Klein (2010), el deseo de abarcar todos estos frentes conlleva el riesgo de ser percibidos como un grupo incoherente de conocimientos por el estudiantado.

De manera que el desarrollo de una visión general sobre la relación entre la ingeniería eléctrica y la inteligencia artificial se encuentra, en cuanto a contenidos, con un doble problema, dada la extensión temática y desarrollo acelerado tanto de la inteligencia artificial como de la ingeniería eléctrica. Si bien los puentes entre ambos son múltiples y se construyen 
continuamente, la selección de contenidos para la relación entre ambas disciplinas debe realizarse con criterios bien establecidos de coherencia y conveniencia.

Una vez que se cuente con una selección de estos contenidos, o bien una guía para que sea el estudiantado quien pueda contar con cierta flexibilidad para abordar algunos temas de acuerdo con sus intereses, es importante también ofrecer herramientas para poner en práctica los conceptos teóricos, idealmente, de una forma contextualizada. Esto, como indican de la Cruz y Rodríguez (2006),

[a]yuda al estudiante a entenderlos de manera más adecuada, ya que le sirve para percibir las ventajas y limitaciones de los diferentes conceptos que se le presentan. Esto es especialmente importante en cursos de introducción a un área, ya que en muchas ocasiones dichos cursos duran poco tiempo en comparación con todos los temas que abarcan. (p.156)

En la literatura se encuentran ejemplos de iniciativas concretas en cursos para la formación en temas afines a la inteligencia artificial en niveles de pregrado de distintas carreras tecnológicas, en los cuales el énfasis principal no está relacionado con la inteligencia artificial. Cuando esto se ha realizado, destaca la aplicación de estrategias novedosas para incorporar los temas y favorecer el aprendizaje del estudiantado. Por ejemplo, en la Universidad California del Sur, Wong, Zink y Koenig (2010, p.1), reportan el uso de videojuegos para el aprendizaje de la inteligencia artificial, ya que los consideran un vehículo de interés donde se pueden aplicar diversos algoritmos de inteligencia artificial (planeamiento de rutas, completar objetivos). Estos autores tienen en cuenta la conveniencia de utilizar una estrategia con varios tipos de proyectos, dada la diversidad de intereses que pueden presentar los y las estudiantes, quienes no necesariamente están enfocados en la inteligencia artificial como opción principal de carrera.

Específicamente con el juego de Pac-Man, DeNero y Klein (2010) han realizado y validado una experiencia en un curso introductorio de inteligencia artificial, en el cual han constatado la motivación generada en el estudiantado ante un problema tan concreto, a partir del cual se pueden relacionar más fácilmente definiciones de problemas más generales.

La idea de utilizar técnicas más allá de la enseñanza tradicional es enfatizada por Chesani, Galassi, Mello y Trisollini (2017, p. 27). Entre estas técnicas se incluyen los juegos y los problemas prácticos en las disciplinas. 
Entre los casos documentados de creación de cursos sobre la temática de inteligencia artificial en carreras de ingeniería, se encuentra la Universidad Tecnológica Nacional-Facultad Regional Santa Fe, en Argentina. En éste,

uno de los objetivos que se persigue es que el alumno sea capaz de aplicar técnicas de IA en la resolución de problemas. Así, desde la asignatura se trata de proveer oportunidades (en forma de situaciones problemáticas a resolver) para que el alumno vea cuáles son los problemas típicos de la IA y cómo éstos pueden ser resueltos desde un punto de vista ingenieril. (Roa, Gutiérrez y Stegmayer, 2008, p. 43)

No existe en estas publicaciones una propuesta específica como la que se discute en el presente ensayo, dirigida a la población que se está formando en el área de ingeniería eléctrica. Esto representa una necesidad ya que una de las funciones principales de las instituciones de educación superior es el formar profesional y promover los cambios que la sociedad necesita. Es sabido que desde la educación se generan elementos vitales no solamente para el desarrollo de una persona en la sociedad y la aplicación de sus eventuales conocimientos en un área como la inteligencia artificial en un contexto particular, sino que también generan recursos importantes para que los países puedan salir adelante a nivel estructural, social y económico (Barrera, López y Bedoya, 2014, p. 7).

Así, la necesidad de formar en elementos de inteligencia artificial a nuevos profesionales debe verse como una respuesta ante el rápido crecimiento e importancia de esta área en los países que no lo han adoptado de forma decisiva, aun cuando existen elementos para su incorporación en un programa de estudio, como el que se analiza en el presente ensayo. Ya que, como indican Valero, Redondo y Palacín (2012):

el conocimiento es el factor clave de la sociedad actual, una sociedad que es el resultado de las enormes transformaciones tecnológicas sucedidas desde finales de los años setenta del siglo pasado. Esta sociedad denominada, no sin controversia, "Sociedad del Conocimiento", se encuentra sometida a constantes cambios y demudaciones debido a la celeridad de los avances tecnológicos. (p. 12)

Estos avances tecnológicos han inclinado cada vez más la balanza en favor de trabajadores calificados, y este factor parece ser la razón de la creciente diferencia salarial entre los individuos calificados y los no calificados en los países industrializados Ferranti, Perry, Gill, Guasch, Maloney, y Sánchez (2003, p. 2). Las industrias actuales, en su necesidad 
de aumentar la productividad y sus capacidades generales, requieren adoptar nuevas tecnologías y para esto también la contratación de trabajadores con mayor calificación en áreas clave, entre las cuales se encuentra sin duda la inteligencia artificial para muchos sectores industriales y de producción tecnológica. La combinación de temáticas de ingeniería eléctrica, ligadas tradicionalmente en un país como Costa Rica a áreas como la producción de energía, a las telecomunicaciones y a la producción de componentes electrónicos y servicios relacionados, y la inteligencia artificial, pueden generar y atraer nuevas oportunidades de desarrollo. Ya que como indican Domínguez, Oliveros, Coronado y Valdez (2019),

la falta de formación profesional es una restricción clave para la innovación, ya que obstaculiza el crecimiento de la productividad y el desarrollo económico, en particular, de aquellos profesionistas y docentes capacitados en las disciplinas relacionadas con la ciencia, tecnología, ingeniería y matemáticas. (p. 16)

Dada la ausencia de propuestas semejantes o recursos didácticos para atender esta necesidad de formación académica en la población contemplada en este ensayo, se considera muy relevante brindar elementos para su construcción, iniciando con los contenidos a considerar.

\subsubsection{Sobre los contenidos temáticos a considerar}

La falta de consenso sobre la definición de inteligencia artificial también se refleja en las divisiones de esta área del conocimiento, y en la selección de aquellos conjuntos de algoritmos y procesos que deben contemplarse en sus alcances. Por ejemplo, en textos introductorios de la materia, Taulli (2019) distingue como sus áreas principales el aprendizaje profundo, la automatización robótica y el procesamiento del lenguaje natural. Por su parte, Ertel (2017) distingue dentro del tema las áreas de Programación Lógica, Problemas de búsqueda y juegos, razonamiento en incertidumbre, aprendizaje de máquina y minería de datos, y finalmente redes neuronales. Flasinski (2016), por su parte, realiza una división más específica a partir de los métodos: Problemas de búsqueda, computación evolutiva, razonamiento lógico, representación del conocimiento, sistemas basados en reglas, reconocimiento de patrones, redes neuronales, razonamiento con conocimiento imperfecto, y arquitecturas cognitivas. Esta división es semejante a la que realiza el texto ampliamente difundido de Rusell y Norvig (2002). Finalmente, cabe mencionar que autores como Borana (2016), incluyen a los sistemas 
expertos, a la lógica difusa y elementos específicos como el test de Turing dentro de las áreas de la inteligencia artificial.

Algunos de estos conceptos pertenecen a dominios específicos del conocimiento que no es posible considerar en un curso introductorio del tema en una carrera de ingeniería eléctrica, ya que guardan una relación distante en relación con los fundamentos necesarios para su comprensión. Tal es el caso del Procesamiento del Lenguaje Natural, el cual está fundamentado en el análisis del texto, o el razonamiento lógico, más cercano a áreas de la matemática.

La selección de contenidos para la consolidación de la incorporación de temáticas relacionadas a la inteligencia artificial en el pregrado de ingeniería eléctrica debe tomar en cuenta también los contenidos propios del área de ingeniería. El acercamiento de generalidades de ambas disciplinas conlleva la gran tarea de abarcar con un nivel de profundidad adecuado elementos tanto de una como de la otra, de manera que se evite dejar por fuera elementos imprescindibles.

Un caso ilustrativo podría ser un curso que no presente técnicas clave dentro de la inteligencia artificial como los métodos de clasificación, por centrarse en predicción de variables y en temas de optimización. Por otra parte, la conjugación de ambas disciplinas en una visión general hace que no sea conveniente dejar por fuera áreas de la ingeniería que sí tienen conexiones relevantes con el tema de inteligencia artificial pero que requieren de una indagación más profunda para establecerlas, como sucede en procesamiento de señales.

En contextos aplicados, las divisiones temáticas dentro de la inteligencia artificial han tenido conexiones claras con la ingeniería eléctrica, tales como los sistemas expertos en robótica (Sanders, Graham-Jones y Gegov, 2010), redes neuronales artificiales (CotoJiménez, 2019; Ekonomou, 2010) y algoritmos evolutivos (Chan, Lee, Sudhoff y Zivi, 2008). Existen referencias previas sobre propuestas para presentar algunos de estos temas utilizando aplicaciones novedosas, como los sistemas expertos presentados por Mariño (2008), quien desarrolló un simulador con una serie de experimentos y material práctico que fomenta también el aprendizaje independiente.

Los mismos autores documentan herramientas de simulación desarrollados para el aprendizaje del tema de redes neuronales artificiales, como la de García, Mariño y Pecorelli (2003). Este tema en particular representa un reto para la comprensión de estudiantes a nivel de pregrado, ya que la gran complejidad de los modelos, así como la diversidad de implementaciones en software que existen, hacen que existe el riesgo de utilizarlas de una 
manera superficial, o bien de profundizar en los algoritmos sin conocer aplicaciones reales o las implementaciones de software.

En el caso de algoritmos genéticos, las posibilidades de utilizar simuladores para su comprensión son presentados por Mariño, (2008), "logrando así una mayor compresión de los conceptos relacionados a esta técnica de la IA" (p. 5).

El desarrollo de estas herramientas es una evidencia de la relevancia de los mismos en contextos de enseñanza más amplios de la inteligencia artificial. Sin embargo, se toman aquí solamente como referencias para destacar la importancia de los contenidos. Además de presentar las redes neuronales, los algoritmos genéticos y los sistemas expertos en contextos lúdicos, un aprendizaje significativo de estos contenidos debe brindar al estudiantado una visión de las conexiones de estos temas con la disciplina de ingeniería eléctrica.

Por estas razones, en un curso introductorio de inteligencia artificial en ingeniería eléctrica se deben hacer esfuerzos por integrar estrategias como estudios de caso y proyectos donde se pueda establecer estas conexiones. Las investigaciones científicas pueden ser una fuente valiosa de información, tanto para su adaptación en la información que se brinda, como en el estudio individual y exploración del estudiantado.

Casos como la aplicación de algoritmos genéticos para la optimización de redes eléctricas (Anaut, Di Mauro, Meschino, y Suárez (2009), sistemas expertos de detección de fallas en calidad de la energía eléctrica (Montoya, Manzano-Agugliaro, López y Alguacil (2012) y el uso de las redes neuronales para predecir el costo de la energía eléctrica (Villada, Cadavid, y Molina, 2008) son ejemplos claros de fuentes de información que se prestan para su adaptación en el nivel de pregrado.

Con estos elementos se puede establecer un conjunto mínimo de contenidos temáticos de inteligencia artificial y su conexión con áreas de la ingeniería eléctrica, establecidos con ejemplos de aplicaciones provenientes de la literatura. Estas relaciones se resumen en la Tabla 1.

Este conjunto mínimo se presenta como un punto de partida para contemplar las principales áreas de ambas disciplinas a partir de las subáreas descritas previamente, y en los cuales se encuentren referencias consolidadas en la literatura reciente asequibles al estudiantado que permitan ilustrar casos de interés. No se pretende establecer una revisión extensiva de referencias, sino ilustrativa para sustentar la selección de los contenidos mínimos para desarrollar una visión general de la relación entre la inteligencia artificial y la ingeniería eléctrica. 
Tabla 1

Conjunto mínimo de contenidos propuestos como necesarios para establecer la relación entre la inteligencia artificial y la ingeniería eléctrica en un curso de pregrado.

\begin{tabular}{|c|c|c|}
\hline $\begin{array}{l}\text { Contenido temático de } \\
\text { inteligencia artificial }\end{array}$ & $\begin{array}{c}\text { Área de relación de } \\
\text { la ingeniería eléctrica }\end{array}$ & Ejemplos de aplicaciones \\
\hline \multirow{3}{*}{ Algoritmos de agrupamiento } & $\begin{array}{l}\text { Procesamiento } \\
\text { señales }\end{array}$ & $\begin{array}{l}\text { - Señales de radar (Matuszewski, } \\
\text { 2018). } \\
\text { - Señales de sonido (Merchan et al., } \\
\text { 2019) }\end{array}$ \\
\hline & Circuitos eléctricos & - Diseño de circuitos (Lin y Liu, 2010). \\
\hline & Electrónica & $\begin{array}{l}\text { - Redes de sensores (Zhang, Xiao, y } \\
\text { Tan, 2013). }\end{array}$ \\
\hline \multirow{2}{*}{$\begin{array}{l}\text { Algoritmos de clasificación } \\
\text { automática }\end{array}$} & Sistemas de energía & $\begin{array}{l}\text { - Predicción de consumo energético } \\
\text { (Wei et al., 2018). }\end{array}$ \\
\hline & Biomédica & $\begin{array}{l}\text { - Análisis de electrocardiogramas } \\
\text { (Hong, Lee, Liang, Hsieh, y Chien, } \\
\text { 2013). }\end{array}$ \\
\hline $\begin{array}{l}\text { Problemas de búsqueda y } \\
\text { computación evolutiva }\end{array}$ & Electrónica & $\begin{array}{l}\text { - Electrónica evolutiva (Zebulum, } \\
\text { Pacheco, y Vellasco, 2018). }\end{array}$ \\
\hline \multirow[t]{2}{*}{ Redes Neuronales Artificiales } & Electromagnetismo & $\begin{array}{l}\text { - Problemas de interferencias (Micu, } \\
\text { Czumbil, Christoforidis, y Simion, } \\
\text { 2012). }\end{array}$ \\
\hline & Telecomunicaciones & $\begin{array}{l}\text { - Sistemas de comunicación (Eriksson, } \\
\text { Bülow, y Leven, 2017). }\end{array}$ \\
\hline
\end{tabular}

Fuente: Elaboración propia, 2021.

\subsubsection{Evaluación}

La diversidad temática y la innovación que requiere la introducción de la inteligencia artificial en una carrera como la Ingeniería Eléctrica conlleva también la necesidad de reflexionar sobre la forma de evaluar el aprendizaje, especialmente si se contemplan implementaciones de algoritmos y casos en software. Como indica de la Cruz y Rodríguez (2005)

El caso de la inteligencia artificial es un ejemplo claro de la dificultad de evaluar a los estudiantes cuando se les enseñan las nociones básicas, como es la solución de problemas usando heurísticas. No basta sólo con determinar si el estudiante conoce las cualidades generales de las diferentes técnicas heurísticas, sino que él debe estar consciente de las limitaciones de cada una de ellas y de los problemas relacionados con su implementación. (p. 150) 
Los mismos autores hacen énfasis en el hecho de que se deben analizar a detalles estas implementaciones, lo cual no es siempre una tarea sencilla por el hecho de que la utilización de la inteligencia artificial conlleva la toma de muchas decisiones en cuanto a la manipulación de los datos, la escogencia de algoritmos y de la gran cantidad de ajustes que existen dentro de los mismos.

Este tipo de dificultades en la evaluación merecen que un curso en esta línea diseñe sus actividades evaluativas con el mismo detenimiento que sus contenidos temáticos, de manera que se favorezca un aprendizaje significativo y no entorpezcan la apreciación de los discentes cuando no son aplicadas de forma correcta. Dado que las fuentes de información actuales sobre la relación entre ambas áreas provienen de artículos científicos, éstos constituyen una posible fuente útil para realizar estudios de caso, replicar o procesar conjuntos de datos, programar los algoritmos o contextualizar los resultados en nuevos entornos.

Como indican los autores de la Cruz y Rodríguez (2005), se debe hacer un énfasis en las limitaciones de las técnicas y algoritmos estudiados, así como de los problemas que surgen en las implementaciones. De manera que estos elementos deben permear los métodos de evaluación propuestos, más allá de la verificación usual del aprendizaje logrado por elementos teóricos o prácticos de los algoritmos en sí.

\subsection{4 Ética}

Uno de los aspectos que hace más interesante el campo de la inteligencia artificial, y la propuesta de permear con ella la formación de profesionales a nivel de pregrado de ingeniería, es que las discusiones éticas son ineludibles, dadas las implicaciones que podría conllevar la aplicación de la inteligencia artificial en contextos más amplios, o incluso de la forma en que se utiliza en la actualidad para el uso de los datos de los usuarios de Internet.

Más bien, como señala Gómez (2016) "los agentes que intervienen en la construcción, uso y manipulación de la IA son quienes por proximidad a la misma, califican y evalúan las posibles implicaciones éticas en su relación con las condiciones humanas." (p.144) Por esto, el estudiantado de ingeniería, quienes son las personas en proceso de convertirse en los agentes de desarrollo de la inteligencia artificial, deben contar desde su primer contacto con la materia con formación en el tema ético.

Como señalan Goldsmith y Burton (2017), la ética en un caso como este tiene dos categorías de decisiones, aquellas tomadas por los profesionales y programadores de los 
sistemas que hacen uso de la inteligencia artificial, y aquellas decisiones tomadas por los algoritmos de inteligencia artificial.

La introducción de estas concepciones éticas puede resultar de utilidad para identificar también formas de pensar en el estudiantado, por ellos mismos, y así abordar la discusión de otros tipos de decisiones. De esta manera, el tratamiento de la ética también puede enmarcarse dentro del aprendizaje significativo, donde "los nuevos conocimientos adquieren significado para el sujeto y los conocimientos previos adquieren nuevos significados o mayor estabilidad cognitiva" (Moreira, 2012, p. 30).

\section{Conclusiones}

En este ensayo se ha realizado un análisis del área de la inteligencia artificial y la necesidad de incorporarla en la formación profesional dentro de un programa de posgrado de Ingeniería Eléctrica. Si bien, el área de la inteligencia artificial surgió como rama independiente del conocimiento en la década de 1950, su estudio dentro de carreras universitarias de pregrado tuvo que esperar mucho más. Ha sido hasta años recientes que se han difundido cursos específicos introductorios del tema en carreras de pregrado, con algunas deficiencias señaladas por diversos autores. Estas deficiencias han empezado a abordarse mediante recursos de innovación, especialmente de simuladores de software.

En el ensayo se ha discutido cómo la integración de la temática en una carrera como ingeniería eléctrica, la cual tiene en su formación los fundamentos teóricos principales para hacerla factible, requiere una profunda reflexión y la incorporación de herramientas tecnológicas para un aprendizaje significativo. Más aún, se ha planteado la conexión con contextos reales, los cuales se pueden encontrar en publicaciones científicas, como una de las fuentes principales de conocimiento útil para establecer, en el estudiantado, el conocimiento, su proyección y aprendizaje de manera significativa.

Estas publicaciones hacen posible evidenciar las aplicaciones de los principales algoritmos de inteligencia artificial en las principales ramas de la ingeniería eléctrica. A partir de esta evidencia, se ha realizado una propuesta inicial de contenidos mínimos que se deben contemplar en un curso introductorio destinado a esta carrera en específico.

Las características del tema, de acuerdo con los elementos analizados en el ensayo, hacen que los aspectos evaluativos deban seguir algunos lineamientos específicos para concienciar al estudiantado de los alcances de los algoritmos en sí y sus contextos de aplicación, además de la ineludible discusión ética. 
La discusión realizada ha mostrado las posibilidades de relacionar la inteligencia artificial con la ingeniería eléctrica a nivel de pregrado, además de evidenciar que existen elementos sobre los que se debe profundizar, como el análisis más extenso en la relación temática entre ambas y las propuestas didácticas específicas para los contenidos. La ausencia de libros de texto publicados específicamente para relacionar ambas áreas llama la atención y se presenta en este contexto como una necesidad. Lo mismo se puede señalar con la ausencia de recursos didácticos, dispositivos y metodologías específicas para el nivel de pregrado en esta disciplina. Todos estos elementos deben someterse a una constante revisión a partir del surgimiento de experiencias concretas de aula y en programas de estudio de carreras de ingeniería.

\section{Referencias}

Anaut, Daniel., Di Mauro, Guillermo, Meschino, Gustavo y Suárez, Juan. (2009). Optimización de redes eléctricas mediante la aplicación de algoritmos genéticos. Información tecnológica, 20(4), 137-148. Recuperado de https://scielo.conicyt.cl/pdf/infotec/v20n4/art16.pdf

Barrera, María., López, Martha., y Bedoya, Martha. (2014). El aprendizaje significativo y la apropiación social de la ciencia y la tecnología. Integralidad educativa. Recuperado de https://aprendizaje-significativo7.webnode.es/ files/200000040694086a38d/EL\%20APRENDIZAJE\%20SIGNIFICATIVO\%20INTEGRALIDAD\%20EDU CATIVA.pdf

Blackshields, Daniel., Cronin, James., Higgs, Bettie., Kilcommins, Shane., McCarthy, Mary., y Ryan, Anthony. (2016). Aprendizaje integrado: Investigaciones internacionales y casos prácticos (vol. 43). Madrid, España: Narcea Ediciones.

Borana, Jatin. (2016). Applications of artificial intelligence \& associated technologies. Presentado en Proceeding of International Conference on Emerging Technologies in Engineering, Biomedical, Management and Science. Recuperado de https://www.cs.buap.mx/ aolvera/IA/2016 Applications\%20of\%20IA.pdf

Chan, Ricky., Lee, Yonggon., Sudhoff, Scott., y Zivi, Edwin. (2008). Evolutionary optimization of powerelectronics based power systems. IEEE Transactions on Power Electronics, 23(4), 1907-1917. Recuperado de https://ieeexplore.ieee.org/document/4558221/authors\#authors

Chesani, Federico., Galassi, Andrea., Mello, Paola., y Trisolini, Giada. (2017). A game-based competition as instrument for teaching artificial intelligence. In Conference of the Italian Association for Artificial Intelligence (pp. 72-84). Springer. Recuperado de https://link.springer.com/chapter/10.1007/978-3-319-70169-1 6 
Choong, Florence., Reaz, M. B.I. y Mohd-Yasin, Faisal. (2005). Advances in signal processing and artificial intelligence technologies in the classification of power quality events: a survey. Electric Power Components and Systems, 33(12), 1333-1349. doi: https://doi.org/10.1080/15325000590964155

Copeland, Jack. (2015). Artificial intelligence: A philosophical introduction. Nueva Jersey, Estados Unidos: John Wiley \& Sons.

Coto-Jiménez, Marvin. (2019). Improving post-filtering of artificial speech using pre-trained LSTM neural networks. Biomimetics, 4(2), 1-39. doi: https://doi.org/10.3390/biomimetics4020039

Das, Sumit., Dey, Aritra., Pal, Akash., y Roy, Nabamita. (2015). Applications of artificial intelligence in machine learning: review and prospect. International Journal of Computer Applications, 115(9), 31-41. doi: http://dx.doi.org/10.5120/20182-2402

De Ferranti, David., Perry, Guillermo., Gill, Indermit., Guasch, Luis., Maloney, William., Sánchez, Carolina., y Schady, Norbert. (2003). Cerrando la brecha en educación y tecnología. Estudio del Banco Mundial sobre América Latina y el Caribe. Recuperado de http://www.launiversidadtecnologicadechile.cl/tportal/portales/tp4964b0e1bk102/uploadl mg/File/TeoriaPractEns/EnsenanzaTecnologia/1 Cerrando brecha entre Ed Tecnolo g.pdf

de la Cruz Martínez, Gustavo., y Rodríguez, Fernando. (2005). Exploración del aprendizaje de los estudiantes haciendo uso de ambientes colaborativos: Enseñando inteligencia artificial. RIED. Revista Iberoamericana de Educación a Distancia, 8(1-2), 147-158. Recuperado de https://www.redalyc.org/pdf/3314/331427204008.pdf

DeNero, Jonh., y Klein, Dan. (2010). Teaching introductory artificial intelligence with pac-man. Presented atln First AAAI Symposium on Educational Advances in Artificial Intelligence. Recuperado de https://www.aaai.org/ocs/index.php/EAAl/EAAl10/paper/view/1954/2331

Domínguez Osuna., Patricia. M., Oliveros Ruiz., María. A., Coronado Ortega, Marcos. A., y Valdez Salas, Benjamín. (2019). Retos de ingeniería: enfoque educativo STEM+A en la revolución industrial 4.0. Innovación educativa (México, DF), 19(80), 15-32. Recuperado de http://www.scielo.org.mx/scielo.php?script=sci arttext\&pid=S1665$\underline{26732019000200015}$

Dorf, Richard. (Ed.). (2018). The Electrical Engineering Handbook-Six Volume Set (3rd ed.). CRC pressNew York: Estados Unidos: Taylor \& Francis.

Ekonomou, Lambros. (2010). Greek long-term energy consumption prediction using artificial neural networks. Energy, 35(2), 512-517. https://doi.org/10.1016/j.energy.2009.10.018

Eriksson, Tobias., Bülow, Henning., y Leven, Andreas. (2017). Applying neural networks in optical communication systems: possible pitfalls. IEEE Photonics Technology Letters, 29(23), 2091-2094. doi: https://doi.org/10.1109/LPT.2017.2755663 
Ertel, Wolfgang. (2017). Introduction to artificial intelligence. New York, Estados Unidos: Springer. Recuperado de https://www.springer.com/gp/book/9783319584867\#aboutBook

Escuela de Ingeniería Eléctrica. (2020). Bachillerato en Ingeniería Eléctrica-Énfasis en Electrónica $y$ Telecomunicaciones. Recuperado de https://eie.ucr.ac.cr/media/planes de estudio/bach-electronica-y-telecomunicaciones2020.pdf

Escuela de Ingeniería Eléctrica. (s.f.). Estudiar en la Escuela de Ingeniería Eléctrica. Recuperado de https://eie.ucr.ac.cr/estudios

Flasiński, Mariusz. (2016). Introduction to artificial intelligence. New York, Estados Unidos: Springer.

García, Lucrecia., Mariño, S.I.. y Pecorelli, Christian. (2003). Entorno virtual de enseñanzaaprendizaje de modelos de redes neuronales artificiales. Comunicaciones Científicas y Tecnológicas. Recuperado de https://studylib.es/doc/8600713/entorno-virtual-deense\%C3\%B1anza---aprendizaje-de-modelos-de-...

Goldsmith, Judy., y Burton, Emanuelle. (2017). Why teaching ethics to Al practitioners is important. In Proceedings of the AAAl Conference on Artificial Intelligence (pp.48364840). Recuperado de https://dl.acm.org/doi/10.5555/3297863.3297908

Gómez, Jairo. (2016). Problemas bioéticos emergentes de la inteligencia artificial. Diversitas: perspectivas en psicología, 12(1), 137-147. Recuperado de http://www.scielo.org.co/pdf/dpp/v12n1/v12n1a11.pdf

Grinsztajn, Fabiana., Imperiale, Marcela., e Igarza, Santiago. (2019). Procesos de Innovación en la Enseñanza de la Ingeniería: La formación por competencias en el DIIT-UNLAM. Presentado en Encuentro Internacional de Educación en Ingeniería. Cartagen, Colombia. Recuperado de https://acofipapers.org/index.php/eiei/article/download/28/23

Han, Yu., y Song, Yong. (2003). Condition monitoring techniques for electrical equipment-a literature survey. IEEE Transactions on Power delivery, 18(1), 4-13. doi: 10.1109/TPWRD.2002.801425

Hong, Jia-Hua., Lee, Shuenn-Yuh., Liang, Ming-Chun., y Chien, Shih-Yu. (2013). A wireless ECG acquisition and classification system for body sensor networks. In 2013 35th Annual International Conference of the IEEE Engineering in Medicine and Biology Society $(E M B C)$ (pp. 5183-5186). Osaka, Japan. Recuperado de https://doi.org/10.1109/EMBC.2013.6610716

Ibrahim, Anis., y Morcos, Medhat. (2002). Artificial intelligence and advanced mathematical tools for power quality applications: a survey. IEEE Transactions on Power Delivery, 17(2), 668-673. doi: 10.1109/61.997958 
Kok, Joost., Boers, Egbert., Kosters, Walter., Van der Putten, Peter., y Poel, Mannes. (2009). Artificial intelligence: definition, trends, techniques, and cases. Artificial intelligence, 1, 120. Recuperado de http://www.eolss.net/sample-chapters/c15/e6-44.pdf

Liao, Weidong., y Guzide, Osman. (2019). Teaching artificial intelligence for undergraduate students: a project based approach. Journal of Computing Sciences in Colleges, 34(3), 132-132. Recuperado de https://dl.acm.org/doi/abs/10.5555/3306465.3306488

Lin, Kupching., y Liu, Lungtien. (2010). U.S. Patent No. 7,747,973. Washington, Estados Unidos: Patent and Trademark Office.

Mariño, Sonia. (2008). Diseño de un entorno virtual de enseñanza aprendizaje para la asignatura de Inteligencia Artificial. Quaderns Digitals, (54). Recuperado de http://www.quadernsdigitals.net/index.php?accionMenu=hemeroteca.VisualizaArticulol $\underline{\text { U.visualiza\&articulo id }=10731}$

Matuszewski, Jan. (2018). Application of Clustering Methods in Radar Signals Recognition. In 2018 International Scientific-Practical Conference Problems of Infocommunications. Science and Technology (PIC S\&T) (pp. 745-751).

Merchan, Fernando., Echevers, Giacomo., Poveda, Héctor., Sanchez-Galan, Javier., y Guzman, Héctor. (2019). Detection and identification of manatee individual vocalizations in Panamanian wetlands using spectrogram clustering. The Journal of the Acoustical Society of America, 146(3), 1745-1757. Recuperado de https://asa.scitation.org/doi/10.1121/1.5126504

Micu, Dan., Czumbil, Levante., Christoforidis, Giorgios., y Simion, Emil. (2012). Neural networks applied in electromagnetic interference problems. Revue Roumain des Sciences Techniques, Serie Electrotechnique et Energetique, 57(2), 162-171. Recuperado de http://revue.elth.pub.ro/upload/419010Art06 \%202 2012.pdf

Miró-Julià, Margaret. (2003). Conceptos básicos en la enseñanza de la Inteligencia Artificial: datos, información y conocimiento. Recuperado de: http://bioinfo.uib.es/ joemiro/aenui/procJenui/Jen2003/miconc.pdf

Montoya, Francsco., Manzano-Agugliaro, Francisco., López, Julio., y Alguacil, Pedro. (2012). Técnicas de investigación en calidad eléctrica: ventajas e inconvenientes. Dyna, 79(173), 66-74. Recuperado de http://www.scielo.org.co/scielo.php?script=sci abstract\&pid=S001273532012000300008\&lng=en\&nrm=iso\&tlng=es

Moreira, Marco. (2012). ¿ Al final, qué es aprendizaje significativo?. Revista Qurriculum, 25, 29-56. Recuperado de https://riull.ull.es/xmlui/handle/915/10652

Mota-Valtierra, Georgina., Rodríguez-Reséndiz, Juvenal., y Herrera-Ruiz, Gilberto. (2019). Constructivism-Based Methodology for Teaching Artificial Intelligence Topics Focused on Sustainable Development. Sustainability, 11(17). doi: https://doi.org/10.3390/su11174642 
Müller, Vincent., y Bostrom, Nick. (2016). Future progress in artificial intelligence: A survey of expert opinion. In Fundamental issues of artificial intelligence (pp. 555-572). New York, Estados Unidos: Springer, Cham.

Rawle, Walter. (2018). The Mathematical Foundations of Artificial Intelligence. Recuperado de https://r1.ieee.org/maine/wp-content/uploads/sites/29/Deep-Conversations-on-DeepLearning09-16-2020.pdf

Real Academia Española. (s.f.). Inteligencia Artificial. En Diccionario de la lengua española. Recuperado de https://dle.rae.es/inteligencia

Roa, Jorge., Gutiérrez, Milagros., y Stegmayer, Georgina. (2008). FAIA: Framework para la enseñanza de agentes en IA. IE Comunicaciones: Revista lberoamericana de Informatica Educativa, 4(7-8), 43-56. Recuperado de https://dialnet.unirioja.es/descarga/articulo/2910864.pdf

Rubio, Clemente., Lermanda, Tomás., Segura, Alejandra., Martínez, Claudia., y Vidal, Christian. (2018). Método para medir la credibilidad de los agentes en videojuegos empleando descripción lingüística de fenómenos complejos y su aplicación como herramienta de apoyo en el proceso de enseñanza-aprendizaje en un curso de inteligencia artificial. In XVIII Conferencia de la Asociación Española para la Inteligencia Artificial (CAEPIA 2018) 23-26 de octubre de 2018 Granada, España (pp. 459-464). Asociación Española para la Inteligencia Artificial (AEPIA). Recuperado de: https://sci2s.ugr.es/caepia18/proceedings/docs/CAEPIA2018 paper 217.pdf

Russell, Stuart., y Norvig, Peter. (2002). Artificial intelligence: a modern approach. Madrid, España: Pearson Education Limited.

Sanders, David., Graham-Jones, Jaspers., y Gegov, Alexander. (2010). Improving ability of tele-operators to complete progressively more difficult mobile robot paths using simple expert systems and ultrasonic sensors. Industrial Robot: An International Journal, 35(5), 431-440.

Recuperado https://www.emerald.com/insight/content/doi/10.1108/01439911011063254/full/html

Simon, Herbert A. (2019). The sciences of the artificial. Cambridge, Estados Unidos: MIT press.

Taulli, Tom. (2019) Artificial Intelligence Basics: A Non-Technical Introduction. California, Estados Unidos: Apress.doi: https://doi.org/10.1007/978-1-4842-5028-0

Valero, Carmen., Redondo, Margarita., y Palacín, Ana. (2012). Tendencias actuales en el uso de dispositivos móviles en educación. La educación digital magazine, (147), 1-21. Recuperado de http://educoas.org/portal/la educacion digital/147/pdf/art unned en.pdf

Villada, Fernando., Cadavid, Diego., y Molina, Juan. (2008). Pronóstico del precio de la energía eléctrica usando redes neuronales artificiales. Revista Facultad de Ingeniería Universidad de Antioquia, (44), 111-118. Recuperado de https://www.redalyc.org/pdf/430/43004412.pdf 
Wei, Yixuan., Zhang, Xingxing., Shi, Yong., Xia, Liang., Pan, Song., Wu, Jinshun., Han, Mengjie., y Zhao, Xiaoyun. (2018). A review of data-driven approaches for prediction and classification of building energy consumption. Renewable and Sustainable Energy Reviews, 82(1), 1027-1047. doi: https://doi.org/10.1016/j.rser.2017.09.108

Wong, Daniel., Zink, Ryan., y Koenig, Sven. (2010). Teaching artificial intelligence and robotics via games. In Proceedings of the EAAl Symposium. Recuperado de http://www.aaai.org/ocs/index.php/EAAl/EAAl10/paper/download/1607/2339

Zebulum, Ricardo., Pacheco, Marco., y Vellasco, Marley. (2018). Evolutionary electronics: automatic design of electronic circuits and systems by genetic algorithms. New York: CRC press.

Zhang, Pengfei., Xiao, Gaoxi., y Tan, Hwee-Pink. (2013). Clustering algorithms for maximizing the lifetime of wireless sensor networks with energy-harvesting sensors. Computer Networks, 57(14), 2689-2704. doi: https://doi.org/10.1016/i.comnet.2013.06.003 
Revista indizada en

scip/o

redalyc.sy latindex

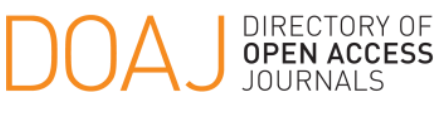

Distribuida en las bases de datos:
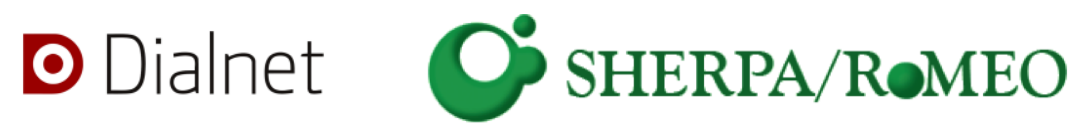

REDIB

Red Iberoamericana

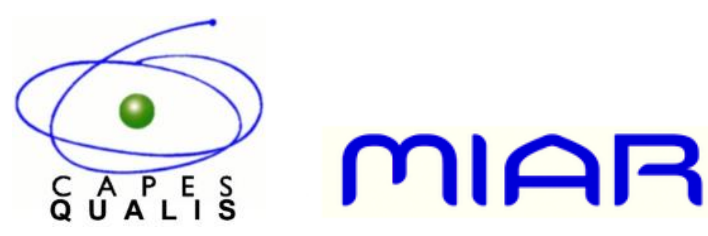

